support for health-related incapacity for work appeared to have little influence on the occurrence of symptoms.

Conclusions There is large international variation in the prevalence of disabling forearm and back pain among occupational groups carrying out similar tasks. This is only partially explained by the personal and socio-economic risk factors that were analysed.

\section{OCCUPATIONAL AND NON-OCCUPATIONAL RISK FACTORS FOR SURGICALLY TREATED CARPAL TUNNEL SYNDROME: PRELIMINARY RESULTS OF A MULTICENTRE POPULATION-BASED CASE-CONTROL STUDY}

${ }^{1} S$ C Curti, ${ }^{1}$ Mattioli, ${ }^{2}$ Baldasseroni, ${ }^{3}$ Bovenzi, ${ }^{1}$ Bonfiglioli, ${ }^{1}$ Violante. ${ }^{1}$ University of Bologna, Bologna, Italy; ${ }^{2}$ Tuscany Regional Centre for Occupational Injuries and Diseases (CeRIMP), Florence, Italy; ${ }^{3}$ University of Trieste, Trieste, Italy

\subsection{6/oemed-2013-101717.253}

Objectives Carpal tunnel syndrome (CTS) is a socially relevant condition that often involves disability. Few large analytical studies have been published considering occupational/non-occupational risk factors. This population-based case-control study aims at investigating both occupational and non-occupational risk factors for surgically treated CTS.

Methods Sixteen centres participated in the study. Each centre identified 200 subjects (aged 25-59 yr): 100 cases (50 women and 50 men) and 100 controls (50 women and 50 men). Cases were randomly drawn from administrative databases of all citizens submitted during 2001 to carpal tunnel release in any public/private hospital with a principal diagnosis of CTS. Controls were randomly sampled from official national health service registry records and were frequency matched by age and gender with cases. Participants were invited by mail (and phone when necessary) to respond to a structured questionnaire regarding occupational (including previous and current job titles along with biomechanical risk factors) and non-occupational risk factors.

Results The sixteen centres overall identified 3,052 subjects (1,458 cases and 1,594 controls) on the base of the study protocol criteria. A total number of 2,294 subjects responded to the questionnaire (1,182 cases and 1,112 controls) corresponding to a response rate of $81 \%$ and $70 \%$ respectively. After exclusion of non-eligible subjects, 1,018 cases and 959 controls entered the main analysis. After adjusting for non-occupational risk factors, manual workers of both sexes appeared to have at least 4-fold risk of surgical treatment of CTS, as compared with non-manual counterparts (women: OR 4.57, 95\% CI 3.07-6.81; men: OR 4.44; 95\% CI 2.96-6.67).

Conclusions This multicentre population-based case-control study strongly underscores the relevance of manual work as an important risk factor for surgically treated CTS, irrespectively of gender. Further analyses will contribute to provide conclusive evidence on the relationship between biomechanical overload and CTS, considering non-occupational risk factors as well.

\section{PSYCHOLOGICAL AND CULTURALLY INFLUENCED RISK FACTORS FOR THE INCIDENCE AND PERSISTENCE OF NON-DISABLING AND DISABLING MUSCULOSKELETAL PAIN. SPANISH CUPID STUDY}

'S V P Vargas-Prada, 'Serra, 'Martínez, ${ }^{2}$ Delclos, ${ }^{3}$ Coggon, 'Benavides. 'Center for Research in Occupational Health (CISAL). Universitat Pompeu Fabra, Barcelona, Spain; ${ }^{2}$ School of Public Health, University of Texas, Houston, United States of America; ${ }^{3} M R C$ Lifecourse Epidemiology Unit, University of Southampton, Southampton, United Kingdom

\subsection{6/oemed-2013-101717.254}

Objective To investigate the role of psychological risk factors for the development and persistence of non-disabling (NDMSP) and disabling (DMSP) musculoskeletal pain (MSP).

Methods As part of the CUPID study, 1105 nurses and office workers were asked at baseline about psychological and workrelated psychosocial risk factors, physical activities in the workplace and MSP in the past month and past year at ten anatomical sites (back, neck, and left and right shoulder, elbow, wrist/ hand and knee). One year later, pain in the past month was again ascertained. Pain was defined as disabling if certain daily activities were difficult or impossible to perform. At baseline, pain-free anatomical sites were included in the analyses for new NDMSP and DMSP, and painful sites in the analysis for persistent NDMSP and DMSP. Analysis was based on anatomical sites and associations were explored using multilevel multinomial logistic regression modelling.

Results 971 participants (87.9\%) completed follow-up. Among 8083 pain-free anatomical sites at baseline, 341 (4.2\%) and 412 (5.1\%) were involved in new NDMSP and DMSP at follow-up. After adjustment for sex, age, occupational risk factors and pain at the same site in the past year, new DMSP was predicted by somatising tendency (OR 3.4, 95\%CI 2.1-5.5). Among 1627 painful sites at baseline, $379(23.3 \%)$ and 500 (30.7\%) still had NDMSP and DMSP one-year later. Having adverse beliefs about the prognosis of MSP was associated with an increased risk of persistence of NDMSP (OR 2.3; 95\%CI 1.2-4.6) and DMSP (OR 2.9; 95\%CI 1.5-5.6).

Conclusion Psychological risk factors may have a stronger role in the development and persistence of DMSP than of NDMSP.

\section{MUSCULOSKELETAL DISORDERS AND PSYCHOLOGICAL COMORBIDITY IN GULF WAR VETERANS AND THE RELATIONSHIP WITH PHYSICAL AND MENTAL HEALTH AND WELLBEING}

L Kelsall, McKenzie, Roberts, Forbes, Urquhart, Sim. Monash University, Melburne, Australia

\subsection{6/oemed-2013-101717.255}

Objective To investigate musculoskeletal disorders, psychological comorbidity, and general physical and mental health and wellbeing in Gulf War veterans and a military comparison group.

Methods Cross-sectional study of 1456 male Australian 1990 1991 Gulf War veterans (veterans) and a non-Gulf comparison group ( $\mathrm{n}=1588$ ). At a medical assessment in 2000-2002, reported doctor diagnosed arthritis or rheumatism, back or neck problems, joint problems, soft tissue disorders were rated by medical practitioners as non-medical, unlikely, possible, or probable diagnoses. Only musculoskeletal disorders rated as probable diagnoses were included in analyses. DSM-IV psychological disorders were measured using the Composite International Diagnostic Interview. Physical and mental health and wellbeing was assessed using the 12-item Short-Form Health Survey (SF-12).

Results Almost one-quarter of veterans (24.5\%) and comparison group $(22.4 \%)$ reported a musculoskeletal disorder (odds ratio OR 1.19 ; 95\% CI 1.00-1.43). Having any or a specific musculoskeletal disorder was associated with depression and 